\title{
Evaluating Notifiable Disease Reporting by Providers: Analysis of Data Element Completeness
}

\author{
Patrick T. Lai ${ }^{\star 1}$, Kavya R. Gujjula1, Shaun J. Grannis ${ }^{2,3}$ and Brian E. Dixon ${ }^{1,3}$ \\ ${ }^{1}$ Indiana University, School of Informatics and Computing, Indianapolis, IN, USA; ${ }^{2}$ Indiana University, School of Medicine, \\ Indianapolis, IN, USA; ${ }^{3}$ Regenstrief Institute, Center for Biomedical Informatics, Indianapolis, IN, USA
}

\section{Objective}

To examine the completeness of data elements required for notifiable disease surveillance from official, provider-based reports submitted to a local health department.

\section{Introduction}

Completeness of public health information is essential for the accurate assessment of community health progress and disease surveillance. Yet challenges persist with respect to the level of completeness that public health agencies receive in reports submitted by health care providers. Missing and incomplete data can jeopardize information reliability and quality resulting in inaccurate disease evaluation and management (1). Additionally, incomplete data can prolong the time required for disease investigators to complete their work on a reported case. Thus, it is important to determine where the scarcity of information is coming from to recognize the characteristics of provider reporting.

\section{Methods}

Data from 1,195 unique patient cases across 7 notifiable diseases were abstracted from official reporting forms (2) and were submitted to a local health department serving a large metropolitan area. The selected diseases were chlamydia, gonorrhea, syphilis, salmonella, histoplasmosis, hepatitis B-acute, and hepatitis C-chronic. Table 1 represents the duration and collection period for each of the selected diseases. Diseases were purposely chosen to represent the broad range managed by local health departments.

A set of data elements consisting of patient, clinical, and provider information was then evaluated for completeness. The level of completeness was determined using a classification method similar to that used by Dixon et al. (3). Fields were considered complete if they contained a value; the recorded value was not validated for accuracy.

\section{Results}

Table 2 depicts the level of completeness for the selected data elements across the targeted diseases. Completeness levels and percentages varied by disease and data element with completeness being higher for patient demographic information (e.g., name, address) than provider demographics (e.g., name, clinic address). The majority of data elements for patient demographics were categorized as mostly to always complete.

\section{Conclusions}

It is important that provider reports are completed in a thorough and timely manner. To increase documentation of provider information, analyses of provider characteristics such as workflow patterns, organizational constraints, and information needs are essential to understand the completeness level of provider information reporting. This will allow us to develop implementation of strategies to increase completeness of reporting across all data elements necessary to assess and investigate notifiable diseases.
Table 1 Duration and collection period of diseases studied

\begin{tabular}{|c|c|c|}
\hline Disease & Duration & Collection Period \\
\hline Chlamydia & 3 months & May 2012 - July 2012 \\
\hline Gonorrhea & 3 months & May 2012 - July 2012 \\
\hline Syphilis & 8 months & December 2011 - July 2012 \\
\hline Histoplasma & 24 months & August 2010 - July 2012 \\
\hline Salmonella & 24 months & August 2010 - July 2012 \\
\hline Hepatitis B-Acute & 24 months & August 2010 - July 2012 \\
\hline Hepatitis C-Chronic & 6 months & February 2012 - July 2012 \\
\hline
\end{tabular}

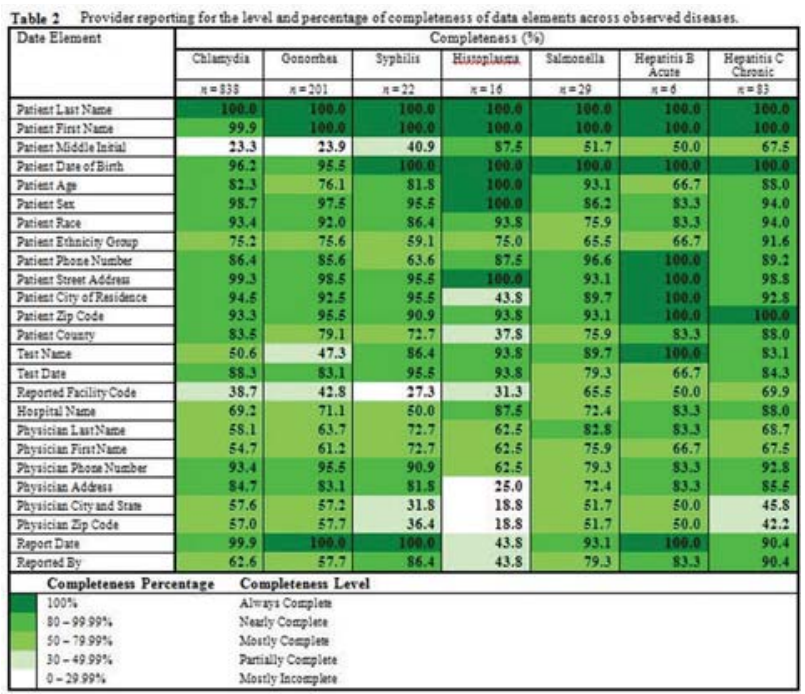

\section{Keywords}

Public Health Surveillance; Disease Notification; Data Quality; Completeness; Communicable Disease Control

\section{Acknowledgments}

This project was supported by grant number R01HS020909 from the Agency for Healthcare Research and Quality. The content is solely the responsibility of the authors and does not necessarily represent the official views of AHRQ.

\section{References}

1) Doyle, TJ, Glynn, MK, Groseclose, SL. Completeness of notifiable infectious disease reporting in the United States: an analytical literature review. Am J Epidemiol. 2002 May 1;155(9):866-74.

2) Indiana Confidential Report of Communicable Diseases-State Form 43823 (R2/11-96) https://forms.in.gov/Download.aspx?id=5082

3) Dixon, BE, Lai, PTS, Grannis, S. Variation in Information Needs and Quality: Implications for Public Health Surveillance and Biomedical Informatics. AMIA Annu Symp Proc. 2013. Forthcoming

*Patrick T. Lai

E-mail: ptlai@imail.iu.edu 